\title{
LA RADIACIÓN SOLAR GLOBAL EN LA PROVINCIA DE LOJA, EVALUACIÓN PRELIMINAR UTILIZANDO EL MÉTODO DE HotTeL
}

\author{
Orlando Álvarez Hernández ${ }^{1, *}$, Thuesman Montaño Peralta ${ }^{2}$ y \\ Jorge Maldonado Correa ${ }^{3}$
}

\section{Resumen}

Existen diferentes modelos teóricos para la obtención de un conocimiento inicial acerca de los valores de radiación solar sobre una superficie horizontal en un lugar determinado. En el presente trabajo, nos enfocamos en la obtención de los valores de radiación sobre una superficie horizontal y se utiliza el conocido "modelo de Hottel". Para la realización del presente trabajo se partió de la confección de un libro de Mi-

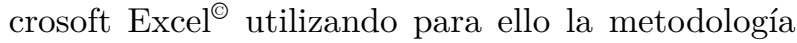
propuesta por Passamai, adicionando un grupo de hojas de cálculo adicionales de forma que se pudiera obtener de forma automática el resumen mensual y anual de los valores diarios para los puntos geográficos utilizados, para poder interpolar posteriormente estos resúmenes en la confección de los diferentes mapas para la zona de trabajo. Un total de 370 puntos fueron procesados de acuerdo a la altura, latitud y longitud correspondientes al MDE, espaciados $0,1^{\circ}$ (aproximadamente cada $10 \mathrm{~km}$ ) obteniéndose los valores horarios, diarios, y a partir de los valores diarios se obtuvieron los mensuales y anuales de radiación directa, difusa y global, sobre una superficie horizontal. Se muestran los valores de la radiación global para cielo claro por meses.

Palabras clave: Radiación solar, modelo Hottel

\begin{abstract}
Different theoretical models exist for obtaining an initial knowledge about the solar radiation values on a horizontal surface in a certain place. The present work is focused in obtaining the solar radiation values on a horizontal surface using Hottel model. For the realization of the present work it was made an Microsoft Excel $^{\odot}$ book and using for it the methodology proposed by Passamai, adding a group of additional worksheets to obtain in an automatic way the monthly and annual summary of the daily values in order to use geographical points to interpolate these summaries later on in a Computer Aid Designs Software (CAD) to made the different maps for the work area. A total of 370 points was processed according to height, latitude and longitude corresponding to the Digital Elevation Model (DEM), spaced $0.1^{\circ}$ (approximately each $10 \mathrm{~km}$ ) to obtained monthly and annual values of direct, diffuse and global radiation, on a horizontal surface. The values of Monthly global radiation are shown for clear sky.
\end{abstract}

Keywords: Solar radiation, Hottel model

\footnotetext{
1,* Doctor en Ciencias Geográficas. Ph.D., Ing. meteorólogo, Investigador Proyecto Prometeo, SENESCYT, Universidad Nacional de Loja, Ecuador. Autor para correspondencia orlando21alvarez@gmail.com

${ }^{2}$ Máster en Ingeniería electromecánica, Ingeniero mecánico, Docente investigador Universidad Nacional de Loja, Ecuador.

${ }^{3}$ Máster en Ingeniería mecánica, Ingeniero electromecánico, Docente investigador Universidad Nacional de Loja, Ecuador.

Recibido: 20-02-2014, Aprobado tras revisión: 26-03-2014.

Forma sugerida de citación: Álvarez, O., Montaño, T. y Maldonado, J. (2014). "La radiación solar global en la provincia de Loja, evaluación preliminar utilizando el método de Hottel". InGENIUs. N. ${ }^{\circ} 11$, (Julio-Diciembre). pp. 25-31. ISSN: 1390-650X.
} 


\section{Introducción}

La tasa a la cual la radiación es recibida por una superficie por unidad de área se denomina irradiancia, la misma que se expresa en unidades de potencia por unidad de área, $\mathrm{W} / \mathrm{m}^{2}$. La cantidad de radiación recibida por una superficie por unidad de área durante un determinado período se denomina irradiación y se expresa en unidades de energía por unidad de área, $\mathrm{Wh} / \mathrm{m}^{2}[1]$.

La radiación solar puede ser estimada para un determinado plano, ya sea horizontal, inclinado o normal a los rayos solares. La radiación solar en plano horizontal representa la radiación solar incidente sobre una superficie horizontal (véase Figura 1).

Si se considera un plano inclinado, la radiación solar representa la radiación solar incidente sobre una superficie inclinada (Figura 2). Por otra parte [1], la radiación solar en plano perpendicular a los rayos solares representa la radiación solar incidente sobre una superficie perpendicular a los rayos solares (Figura 3).

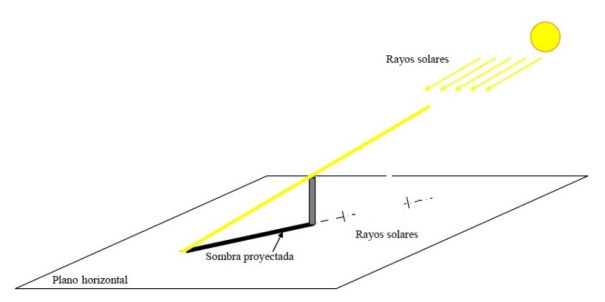

Figura 1. Radiación solar sobre un plano horizontal.

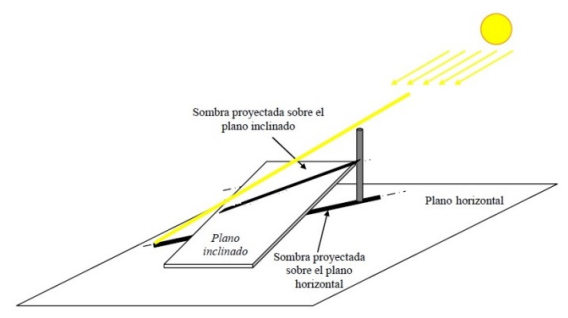

Figura 2. Radiación sobre un plano inclinado.

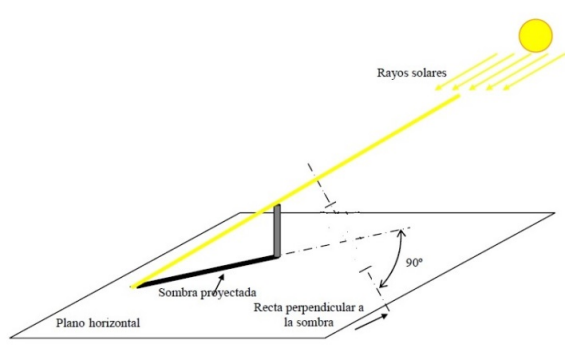

Figura 3. Radiación sobre una superficie perpendicular a los rayos solares.
Existen diferentes modelos teóricos para la obtención de un conocimiento inicial de los valores de radiación solar sobre una superficie horizontal en un lugar determinado, entre los cuales se pueden mencionar los de Orgill y Hollands, Page, Iqbal, Liu y Jordan, ASHARE y Hottel [2].

Obviamente es necesario determinar, mediante los datos obtenidos de radiación directa y global, los instrumentos adecuados para la medición de dichas radiaciones para un lugar señalado, cuál o cuáles de ellos tienen un mejor comportamiento para realizar su respectivo ajuste y de esta manera escoger el o los que ofrecen mayor precisión. En el presente trabajo, solamente nos enfocaremos en la obtención de los valores de radiación sobre una superficie horizontal utilizando el conocido "modelo de Hottel" [3].

Se conoce que la atmósfera ejerce un efecto de redistribución de la radiación solar. Por ejemplo, en un día muy despejado, una parte relativamente pequeña se convierte en radiación difusa, mientras que la mayor parte permanece como directa. En cambio, en un día nublado, la redistribución de la radiación es mucho más notable. Las nubes densas tienen un albedo (fracción de energía reflejada) muy alto, lo cual hace que, en un día densamente nublado, gran parte de la radiación solar se refleje al espacio exterior; la energía que logra pasar a través de las nubes, es únicamente radiación difusa.

Es muy difícil desarrollar modelos para predecir con precisión la presencia de nubes (posición, densidad, etc.). Existen algunos para predicción de "días promedio" en cierta fecha, pero no para fechas específicas. En cambio, existe una diversidad de modelos para estimar la radiación solar para días despejados. Básicamente, estos modelos aplican un factor de transmitancia a la radiación extraterrestre.

Para períodos de una hora (no para todo el día), es posible también usar el mismo factor de transmitancia para estimar la irradiación en un día despejado. El problema consiste en el cálculo o la estimación de la transmitancia atmosférica para la radiación directa, $\tau_{b}$ y para la difusa, $\tau_{d}$.

Entonces, el procedimiento para la utilización de este modelo atmosférico es, una vez dadas la latitud geográfica, la fecha y la hora, calcular el coseno del ángulo cenital, según lo expuesto anteriormente; después computar los valores de la transmitancia directa y difusa, y aplicar los mismos para períodos de una hora.

\section{Materiales y métodos}

Para realizar este trabajo se partió con la elaboración de un libro Excel usando la metodología propuesta por Passamai [4], adicionando un grupo de hojas de cálculo 
de forma que se pueda obtener automáticamente el resumen mensual y anual de los valores diarios para los puntos geográficos considerados, y luego interpolar estos resúmenes para confeccionar los diferentes mapas de la zona de trabajo.

Este modelo expresa la transmitancia atmosférica $\tau_{b}$, en función del ángulo cenital $\theta_{z}$, de la altura sobre el nivel del mar $A$ y del tipo de clima $\left(r_{1}\right)$. El modelo de Hottel [4] es de la forma:

$$
\tau_{b}=a_{0}+a_{1} \cdot \mathrm{e}^{-k / \cos \theta_{z}}
$$

Aquí $a_{0}, a_{1}$ y $k$ son parámetros ajustados empíricamente [4]. Para el cálculo de estas cantidades, que viene siendo la corrección por altura y tipo de clima, se usan las ecuaciones:

$$
\begin{aligned}
& a_{0}=r_{0}\left[0,4237-0,00821(6-A)^{2}\right] \\
& a_{1}=r_{1}\left[0,5055+0,00595(6,5-A)^{2}\right] \\
& k=r_{k}\left[0,2711+0,01858(2,5-A)^{2}\right]
\end{aligned}
$$

El valor $A$ en estas ecuaciones representa la altura sobre el nivel del mar, en kilómetros. Los valores de $r_{1}$ están dados en la Tabla 1, para diversos tipos de clima. En nuestro caso se consideró como verano de latitud media.

\begin{tabular}{lccc}
\hline Tipo de clima & $r_{0}$ & $r_{1}$ & $r_{k}$ \\
\hline Tropical & 0,95 & 0,98 & 1,02 \\
Verano, latitud media & 0,97 & 0,99 & 1,02 \\
Verano, subártico & 0,99 & 0,99 & 1,01 \\
Invierno, latitud media & 1,03 & 1,01 & 1,00 \\
\hline
\end{tabular}

Tabla 1. Factores de corrección para algunos tipos de climas.

Para la utilización de este modelo atmosférico, dadas la latitud geográfica, la fecha y la hora, debe calcularse el coseno del ángulo cenital, según la siguiente ecuación:

$$
\cos \left(\theta_{z}\right)=\operatorname{sen}(\phi) \operatorname{sen}(\delta)+\cos (\phi) \cos (\delta) \cos (\omega)
$$

Donde

$\phi$ : latitud geográfica

$\omega$ : ángulo horario (por definición $\omega=0$ al mediodía solar)

$\delta$ : declinación solar, calculada mediante la ecuación:

$$
\delta=23,45 \operatorname{sen}\left(2 \pi \frac{284+n}{365}\right)
$$

\section{Donde}

$n$ : día del calendario juliano

Para estimar la irradiación o la irradiancia difusa sobre una superficie horizontal se utiliza la expresión de Liu y Jordan (1960) de la forma:

$$
\tau_{d}=0,2710-0,2939 \tau_{b}
$$

Integrando estas ecuaciones, desde la salida hasta la puesta del Sol, se obtiene la irradiación a lo largo de un día. En el modelo de Hottel, la atmósfera estándar representa una condición típica de latitud media y sin contaminación por polución, independientemente del grosor del ozono [2].

Se utilizaron los datos de elevación obtenidos del Digital Elevation Model (DEM) para el mundo, confeccionado a partir de información satelital por la Agencia de Geofísica de los Estados Unidos, incluido en la mayoría de los Sistemas de Información Geográfica (SIG) y en específico en el SIG ENVI 4.0.1, el cual fue cortado y la información de longitud, latitud, alturas mínima, máxima y promedio fueron introducidas en el CAD SURFER, obteniéndose por interpolación, cada $0,1^{\circ}$ de longitud y latitud, el valor correspondiente a la altura máxima en cada punto como se muestra en la Figura 4.

Un total de 370 puntos fueron procesados de acuerdo a la altura, latitud y longitud correspondientes al MNT, espaciados $0,1^{\circ}$ (aproximadamente cada $10 \mathrm{~km}$ ) obteniéndose los valores horarios y diarios; a partir de estos últimos se obtuvieron los mensuales y anuales de radiación directa, difusa y global sobre una superficie horizontal.

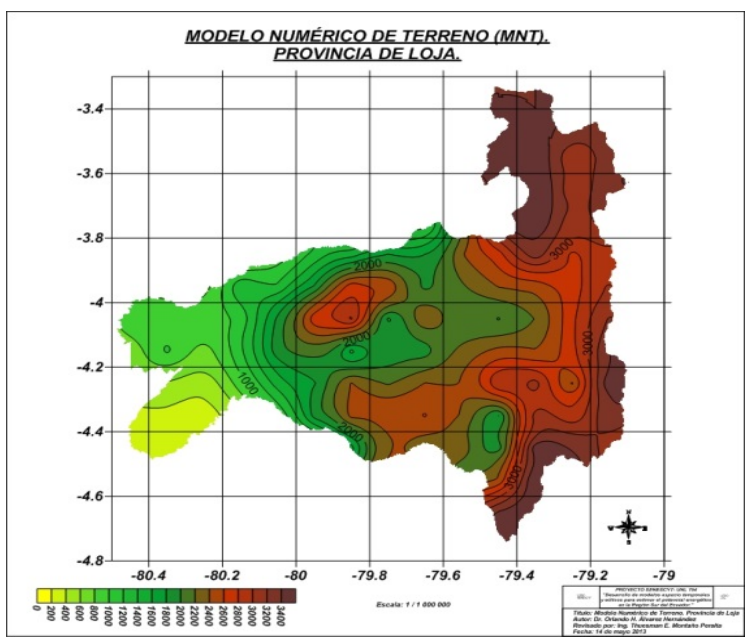

Figura 4. Modelo numérico de terreno de la provincia de Loja. 


\section{Resultados y discusión}

Los valores de la radiación global, por meses, se muestran en las figuras 5 - 16 .

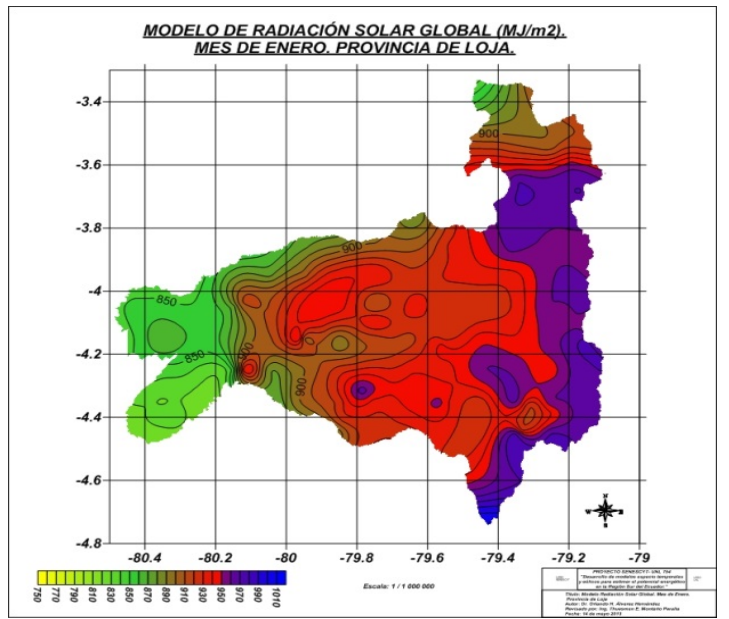

Figura 5. Radiación global, mes de enero.

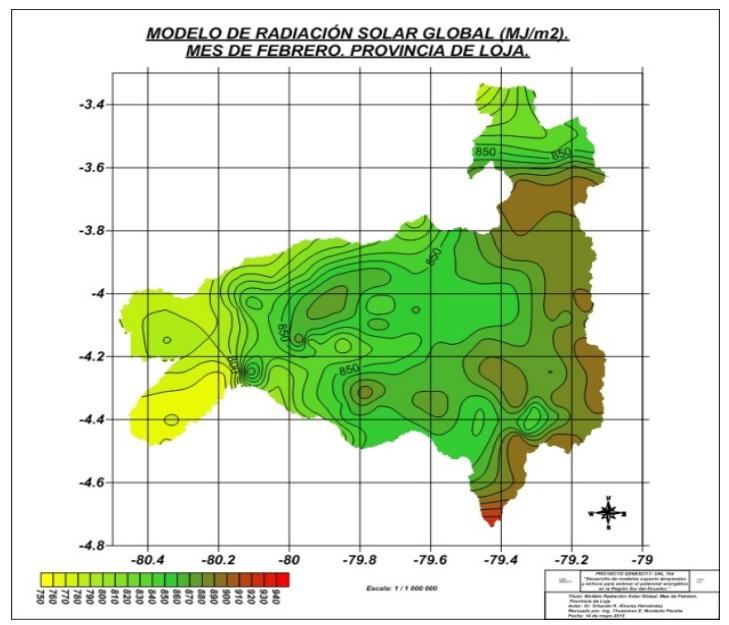

Figura 6. Radiación global, mes de febrero.

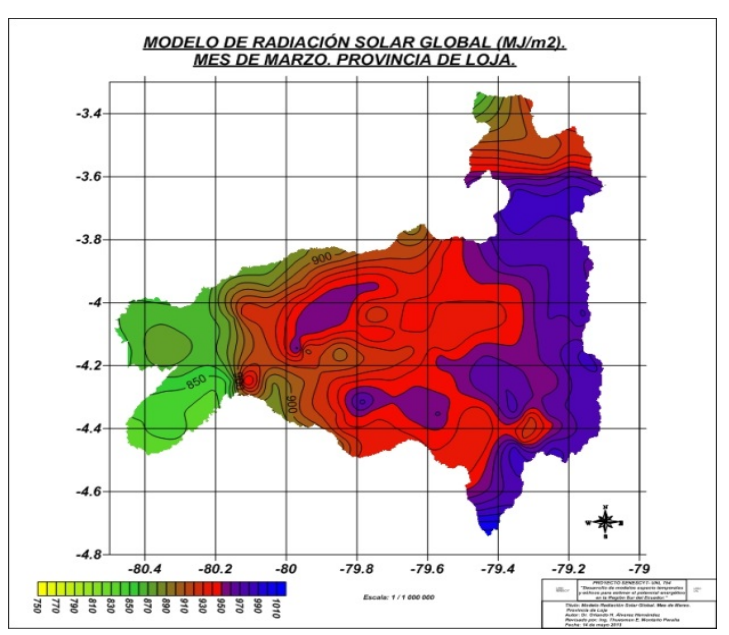

Figura 7. Radiación global, mes de marzo.

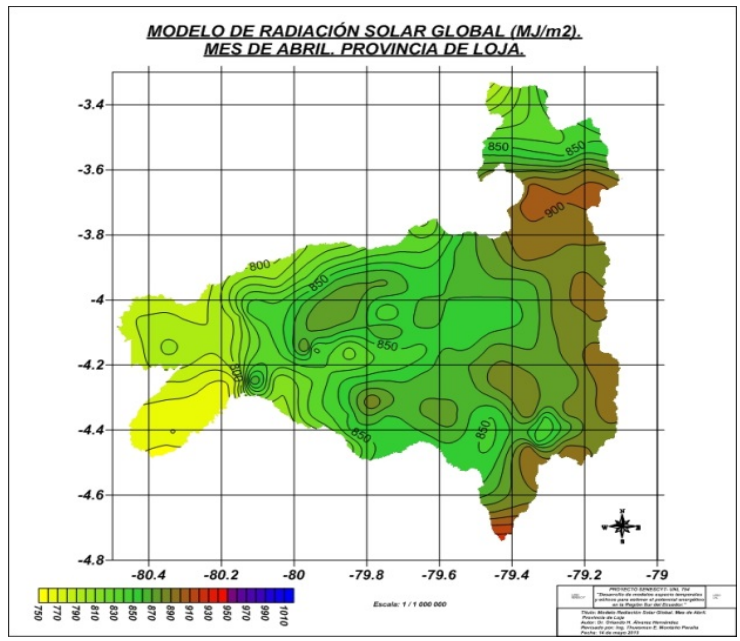

Figura 8. Radiación global, mes de abril.

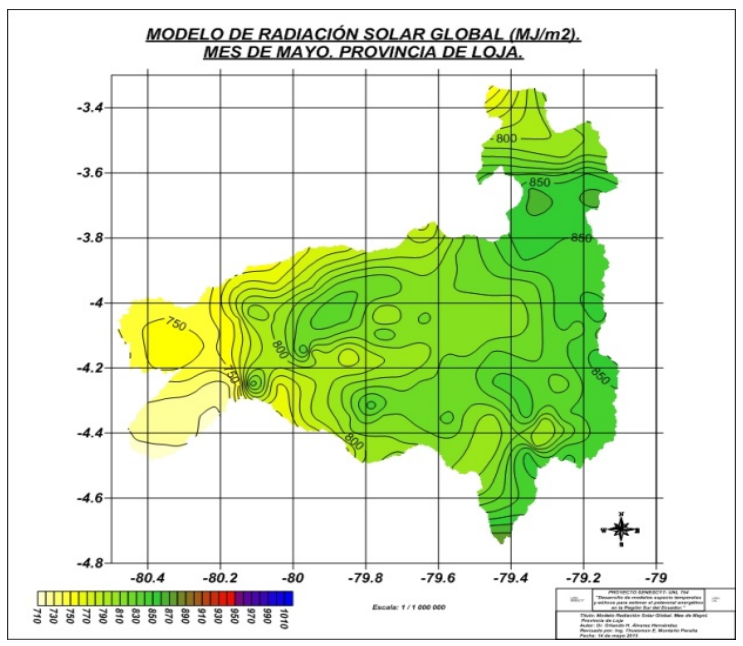

Figura 9. Radiación global, mes de mayo.

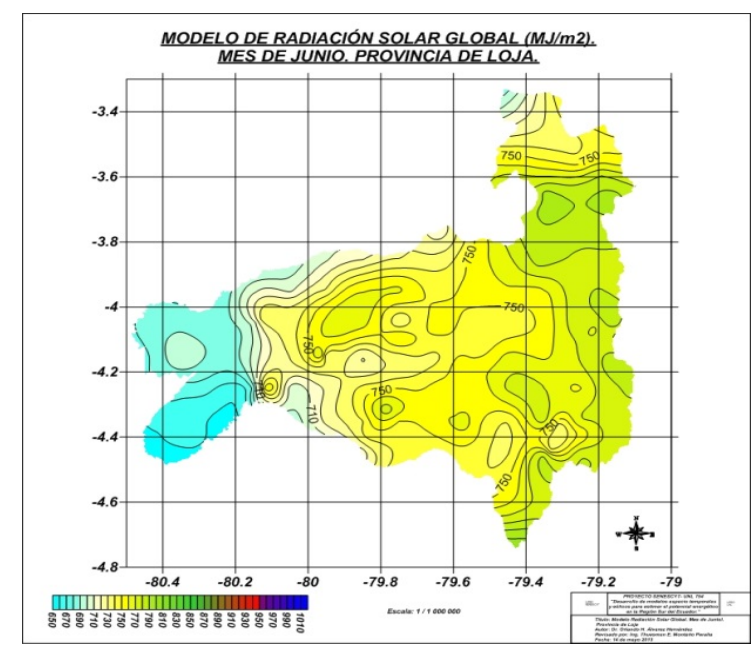

Figura 10. Radiación global, mes de junio. 


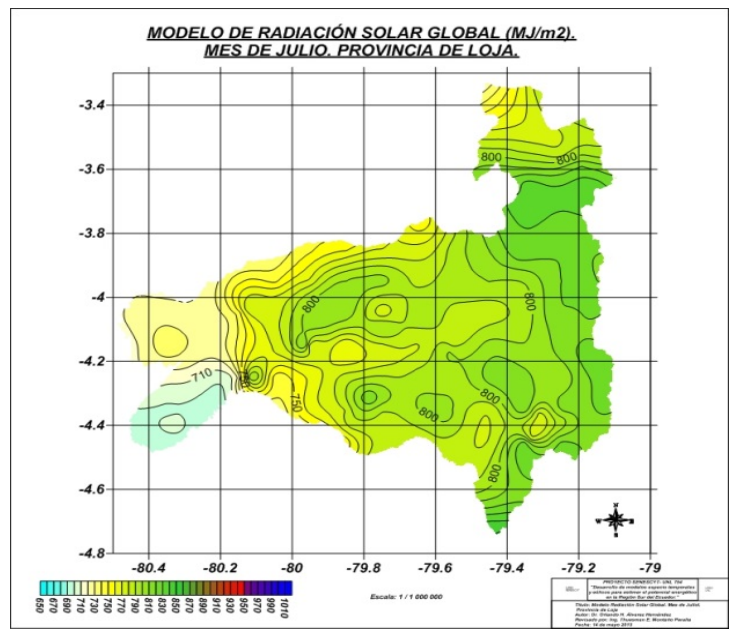

Figura 11. Radiación global, mes de julio.

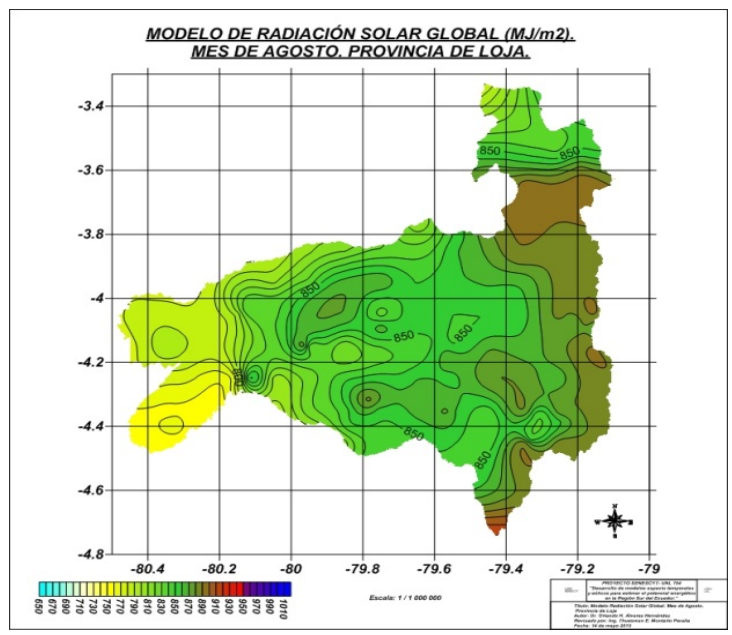

Figura 12. Radiación global, mes de agosto.

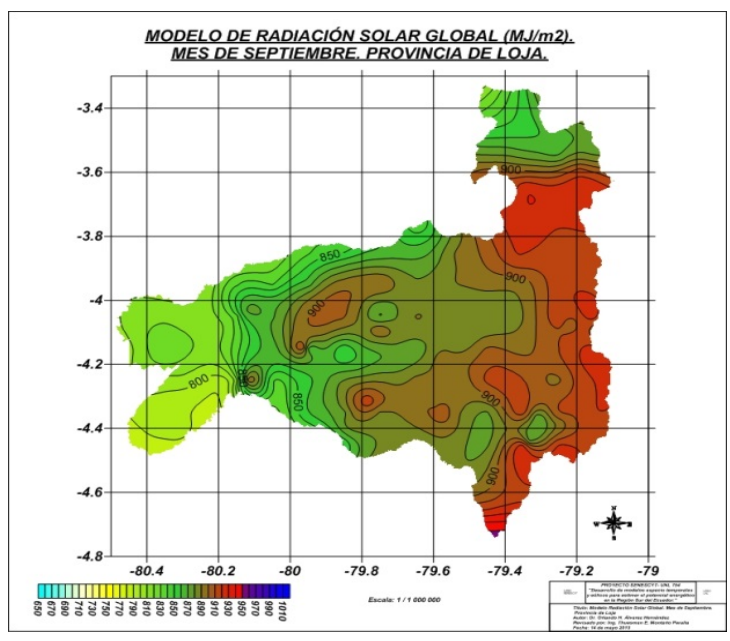

Figura 13. Radiación global, mes de septiembre.

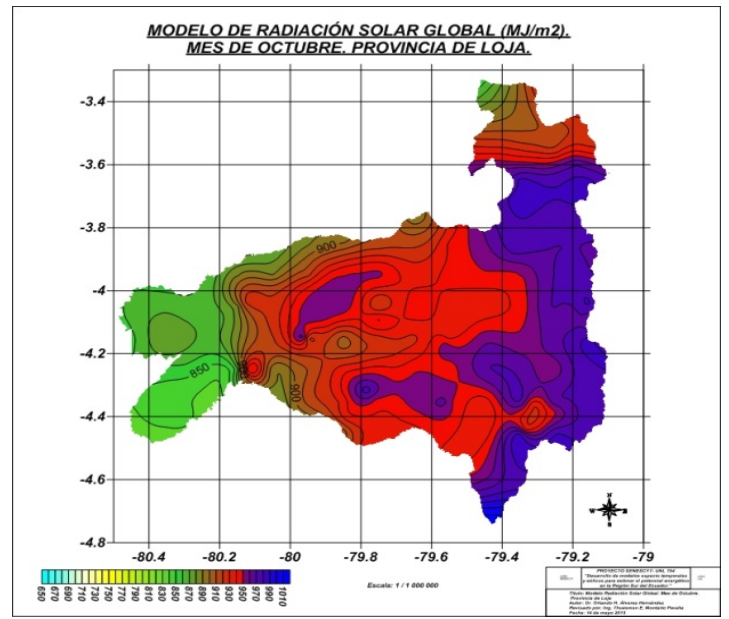

Figura 14. Radiación global, mes de octubre.

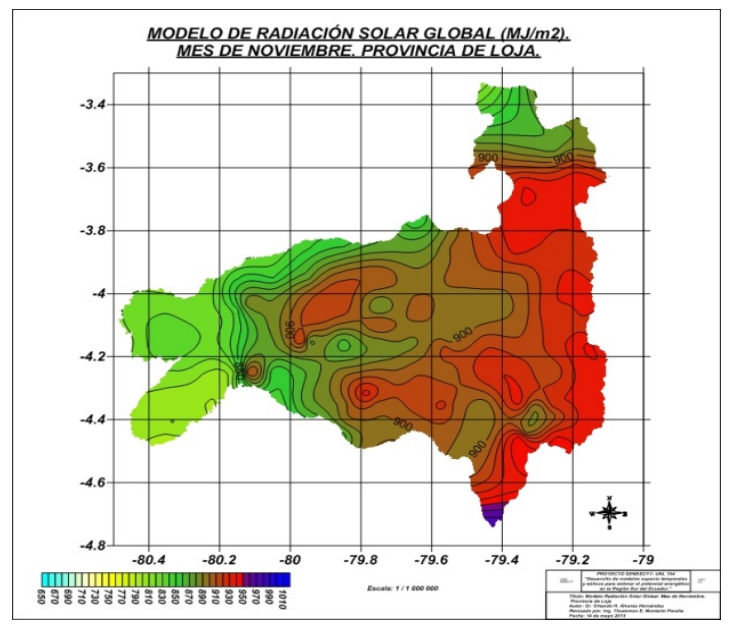

Figura 15. Radiación global, mes de noviembre.

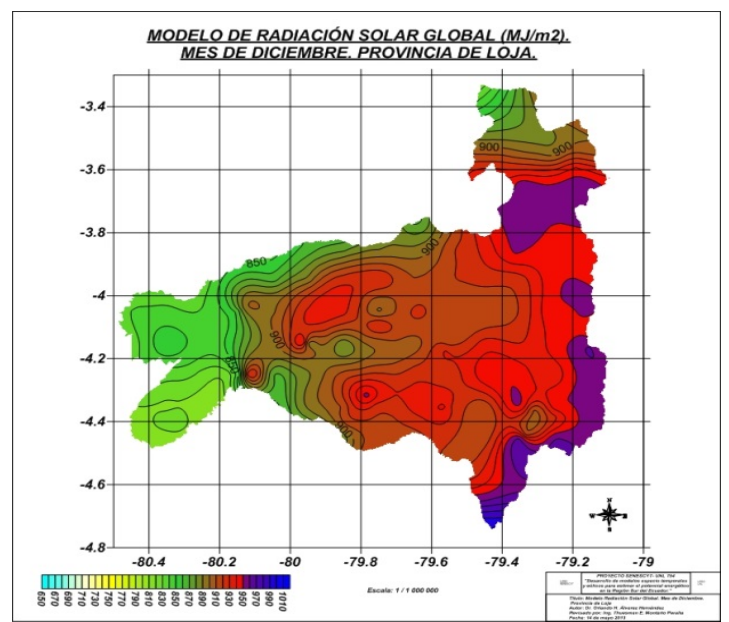

Figura 16. Radiación global, mes de diciembre.

En la Figura 19 se muestra el percentil 50 para los valores diarios en los diferentes meses, pudiéndose observar que los mínimos corresponden al mes de junio, 
Tabla 2. Valores de los estadígrafos para la radiación global.

\begin{tabular}{|c|c|c|c|c|c|c|c|c|c|c|c|c|c|c|}
\hline & I & II & III & IV & $\mathrm{V}$ & VI & VII & VIII & IX & $\mathrm{X}$ & $\mathrm{XI}$ & XII & Annual & Suma año \\
\hline Minimo & 822,96 & 759,52 & 832,57 & 757,13 & 714,48 & 653,08 & 609,67 & 750,28 & 783,54 & 833,71 & 797,21 & 812,37 & 767,29 & 9207,50 \\
\hline Maximo & 1013,89 & 930,81 & 1016,99 & 927,04 & 880,39 & 808,24 & 852,94 & 920,60 & 957,21 & 1020,17 & 980,72 & 1002,65 & 942,68 & 11312,12 \\
\hline Media & 920,90 & 849,26 & 932,17 & 851,82 & 809,25 & 742,75 & 783,93 & 846,15 & 878,45 & 932,33 & 891,82 & 909,50 & 862,36 & 10348,32 \\
\hline Varianza & 1427,73 & 1166,70 & 1383,33 & 1203,45 & 1172,71 & 1038,00 & 1128,81 & 1219,39 & 1237,99 & 1391,90 & 1323,47 & 1415,35 & 1251,40 & 18203,39 \\
\hline Desv. Est & 37,79 & 34,16 & 37,19 & 34,69 & 34,24 & 32,22 & 33,60 & 34,92 & 35,19 & 37,31 & 36,38 & 37,62 & 35,38 & 424,50 \\
\hline Rango interc. & 45,72 & 40,84 & 43,53 & 39,21 & 37,76 & 35,17 & 36,84 & 39,06 & 40,73 & 44,33 & 43,91 & 45,67 & 40,86 & 490,31 \\
\hline Rango & 190,94 & 171,29 & 184,43 & 169,91 & 165,90 & 155,15 & 162,27 & 170,31 & 173,68 & 186,46 & 183,51 & 190,28 & 175,39 & 2104,62 \\
\hline Coef de varian. & 0,04 & 0,04 & 0,04 & 0,04 & 0,04 & 0,04 & 0,04 & 0,04 & 0,04 & 0,04 & 0,04 & 0,04 & 0,04 & 0,04 \\
\hline Asimetria & $-0,85$ & $-0,86$ & $-0,88$ & $-0,91$ & $-0,92$ & $-0,93$ & $-0,93$ & $-0,91$ & $-0,89$ & $-0,87$ & $-0,85$ & $-0,84$ & $-0,89$ & $-0,89$ \\
\hline Curtosis & 2,95 & 2,99 & 3,06 & 3,16 & 3,23 & 3,27 & 3,25 & 3,19 & 3,10 & 3,01 & 2,96 & 2,93 & 3,09 & 3,09 \\
\hline
\end{tabular}

mientras los máximos ocurren en octubre y marzo, lo cual concuerda con los valores de los estadígrafos de la Tabla 2.

También se destaca que, independientemente de la altura máxima que el Sol alcance sobre el horizonte, la distribución de montos de energía recibida por los sectores de superficie incluso cercanos, no son iguales, debido a su pendiente y orientación.

De igual forma se calcularon los valores promedio y sumario anuales (Figuras 17 y 18).

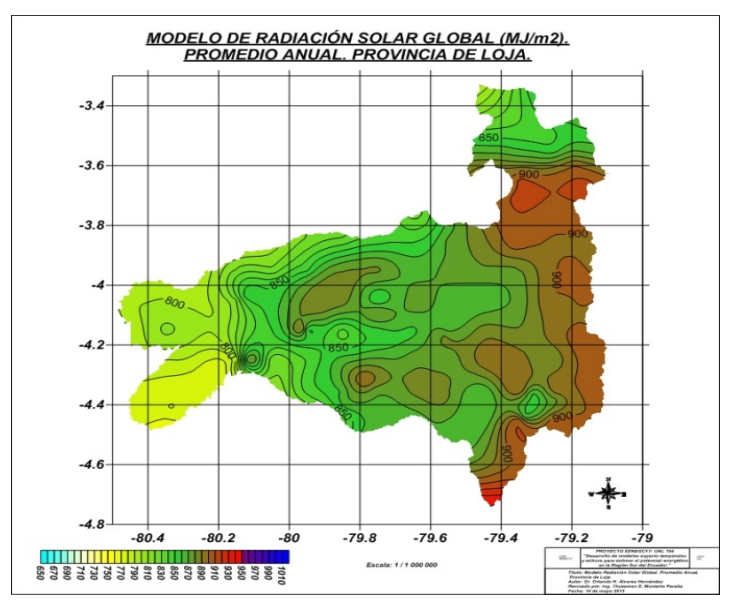

Figura 17. Radiación global. Promedio anual.

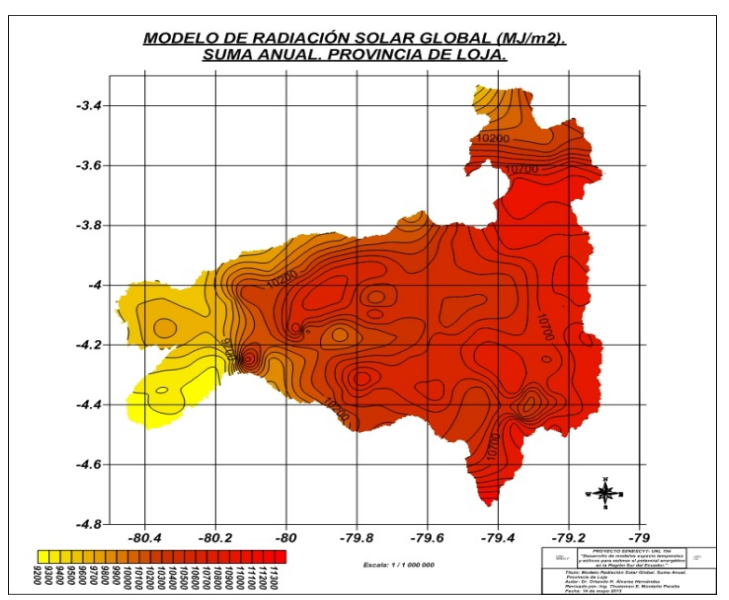

Figura 18. Radiación global. Suma anual.

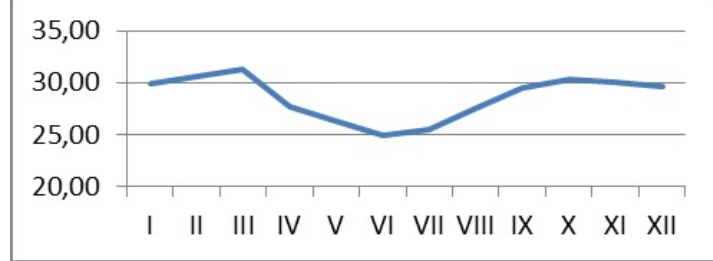

Figura 19. Valores medios diarios. Percentil 50\%.

\section{Conclusiones}

Los valores máximos de radiación global bajo condiciones de cielo claro se encuentran al este de la provincia de Loja y en otras zonas con valores altos de elevación del terreno sobre el nivel del mar, mientras los mínimos aparecen en los sectores más bajos, fundamentalmente hacia el oeste de la provincia de Loja.

En el estudio se demuestra que los meses de octubre y marzo presentan los valores más elevados de radiación global en condiciones de cielo despejado, mientras junio es el mes que recibe menor cantidad de radiación global.

Los valores deben ser menores considerando la nubosidad media para cada mes y año, así como aquellos de pendiente y orientación; sin embargo, se establece una primera aproximación a la radiación solar global en la provincia de Loja.

\section{Recomendaciones}

Aplicar al presente modelo correcciones por la nubosidad, pendiente y orientación de las zonas estudiadas.

Realizar los mismos cálculos utilizando otros modelos que permitan comparar el o los más apropiados para las condiciones topográficas del Ecuador.

Comparar los diferentes modelos con valores medidos en las estaciones automáticas de las cuales ya se posea información. 


\section{Agradecimientos}

Este trabajo científico ha sido financiado por el Proyecto Prometeo de la Secretaría Nacional de Ciencia, Tecnología e Innovación (Ecuador).

\section{Referencias}

[1] S. Guevara, "Estimación de la radiación solar," UNATSABAR-OPS/CEPIS/03, Lima, 2003.

[2] C. Sánchez, D. Piedra, and I. Mendoza, "Validación y ajuste de modelos de radiación solar directa para la ciudad de Bogotá a partir de datos experimentales tomados en la Universidad Distrital Francisco José de Caldas," Revista Colombiana de Física, vol. 38, no. 4, pp. 1435-1438, 2006.

[3] H. C. Hottel, "A simple model for estimating the transmittance of direct solar radiation through clear atmospheres," Solar Energy, vol. 18, no. 2, pp. $129-134,1976$.

[4] V. Passamai, "Determinación de radiación solar horaria para días claros mediante planilla de cálculo," Avances en Energías Renovables y Medio Ambiente, 2000. 